\title{
Dependencia mediática y evaluación de la gestión gubernamental mexicana de la pandemia
}

\section{Media dependency and evaluation of Mexican governmental administration of pandemic}

Carlos Muñiz* (D) http://orcid.org/0000-0002-9021-8198

Universidad Autónoma de Nuevo León, México, carlos.munizm@uanl.mx

Victoria Isabela Corduneanu (10) http://orcid.org/0000-0001-7958-2944

Universidad Autónoma de Ciudad de México, México, isabela.corduneanu@uacm.edu.mx
${ }^{*}$ Autor para correspondencia : Carlos Muñiz, carlos.munizm@uanl.mx

Recepción: 22/02/2021

Aprobación: 04/11/2021

Publicación: 08/12/21

\begin{abstract}
During a crisis context such as the Covid-19 pandemic, the population's dependence on the media system to seek information and guidance increases, which can generate and/or increase political attitudes, such as support for the governmental administration of the crisis. In particular, the article seeks to determine if media dependence influenced the citizen evaluation of the management of the president and the subsecretary of Health during the pandemic in Mexico and if the level of political partisanship moderated this effect. A survey with a representative sample at national level $(N=1211)$ was carried out. Findings allow observing a moderating effect of partisanship on the relationship between dependency and the evaluation of pandemic administration, especially in the case of the subsecretary of Health. This suggests that the differentiated media coverage of both political actors contributed to modifying the citizen evaluation, especially among nonpartisans.
\end{abstract}

Key words: media system dependency, governmental administration evaluation, partisanship, pandemic.

Resumen: En contextos de crisis como la pandemia por Covid-19 se incrementa entre la población la dependencia del sistema mediático para buscar información y orientación, lo que puede generar y/o acrecentar actitudes políticas, como el apoyo a la gestión gubernamental de la crisis. Así, el presente artículo busca determinar si la dependencia mediática influyó en la evaluación ciudadana de la gestión del presidente y del subsecretario de Salud durante la pandemia en México y si el nivel de partidismo moderó este efecto. Para ello, se realizó una encuesta $(N=1211)$ con una muestra representativa a nivel nacional. Los resultados muestran un efecto moderador del partidismo sobre la relación entre la dependencia y la evaluación de la gestión de la pandemia, principalmente en el caso 
del subsecretario de Salud. Ello sugiere que la cobertura mediática diferenciada de ambos actores políticos contribuyó a modificar la evaluación ciudadana, en especial entre quienes eran apartidistas.

Palabras clave: dependencia del sistema mediático, evaluación de la gestión gubernamental, partidismo, pandemia.

\section{Introducción $^{1}$}

Cuando el 11 de marzo de 2020 la Organización Mundial de la Salud (OMS) declaró la pandemia mundial debido a la enfermedad por coronavirus (Covid-19), los diferentes gobiernos nacionales comenzaron a tomar decisiones de actuación respecto a sus países. En el caso de México, el 23 de marzo el gobierno federal declaró la Jornada Nacional de Sana Distancia, buscando el confinamiento voluntario de la población y el mantenimiento de la distancia social en lugares públicos. A partir de esa declaración, la actividad administrativa del gobierno se vio ampliamente reducida, enfocándose buena parte de la estrategia comunicativa institucional en la transmisión diaria de información relativa a la pandemia a la población, a través de diferentes conferencias de prensa desarrolladas por el presidente y el subsecretario de Salud. Esta situación se mantuvo hasta julio de 2020, cuando se emprendió el retorno a la normalidad del país. A partir de este momento, el discurso público realizado por el gobierno se ha centrado en la vertiente más política de la pandemia, aunque siempre manteniendo como una constante la apelación a los "especialistas" y el necesario seguimiento de sus recomendaciones.

En situaciones como la descrita, que supone un escenario de amenaza para la salud pública, pero también durante una crisis nacional e internacional en diferentes niveles (como el social, económico, político, etc.), tiende a incrementarse entre la población el consumo y exposición a los medios de comunicación como mecanismo de búsqueda de información y orientación sobre cómo actuar (Farré, 2005; Garfin et al., 2020; Morton y Duck, 2001; Muñiz, 2011). Esta relación entre público y medios en momentos de crisis e incertidumbre ha sido explicada desde la teoría de la dependencia del sistema mediático (Ball-Rokeach y DeFleur, 1976), la cual sostiene que la ciudadanía amplía su vinculación respecto de los medios de comunicación como fuentes primarias de información durante momentos en que tienen lugar conflictos

$1 \mathrm{El}$ presente artículo fue desarrollado en el marco del proyecto de investigación titulado "Análisis de la cobertura mediática de la pandemia de COVID-19 en México y de su impacto en el desarrollo de actitudes y comportamientos entre la ciudadanía” (clave 312437), financiado por Conacyt. 
dentro del sistema social, ya sean como resultado de desastres naturales, de salud pública o debido a decisiones y acciones humanas (Hindman, 2004; Lin et al., 2020; Morton y Duck, 2001; Muñiz, 2011).

Aunque la contingencia por Covid-19 es relativamente reciente, ya existe evidencia acerca de cómo la exposición a medios realizada durante la pandemia tendió a incrementarse (Duc Huynh, 2020). Por ejemplo, CaseroRipollés (2020) ha detectado en su reciente estudio cómo en Estados Unidos el consumo de medios aumentó entre la población conforme la pandemia transcurría y afectaba a ese país. Pero no solamente es esperable que la ciudadanía obtenga información en los medios para decidir cómo actuar ante una situación de crisis como la pandemia. También es importante la labor que los medios hacen dando a conocer la gestión política que los gobiernos realizan, por ejemplo, ante una crisis (Davies, 2009). Y es que, aunque muchos ciudadanos no demuestran habitualmente interés y atracción por la política, es posible que en contextos de cambio y conflicto su atención mediática aumente, formando gracias a ello una opinión acerca de la actuación de los gobernantes para abordar la crisis (Donovan et al., 2020).

En este sentido, la investigación previa ha podido detectar que la dependencia mediática tiende a generar y/o incrementar actitudes de corte político (Davies, 2009; Halpern, 1994; Ognyanova y Ball-Rokeach, 2015), como puede ser el caso del apoyo a gobernantes durante las crisis. Al respecto, Hindman (2004) detectó una clara relación entre la dependencia y el apoyo a la actuación del presidente de Estados Unidos durante el ataque del 11-S de 2001, la cual estuvo moderada por el partidismo de los ciudadanos. Situaciones como la antes descrita constituyen sucesos imprevistos que escapan a los planes y la programación política que suelen realizar los gobiernos, afectando la popularidad de los diferentes actores políticos involucrados en el mismo (Canel, 2009). Por ello, y teniendo en cuenta que la "popularidad es un recurso político vital" (Krosnick y Kinder, 1990: 497) que se expresa en un alto nivel de atención mediática (Todhunter, 2013), es de esperar que la cobertura de la gestión ante una crisis influya en el apoyo manifestado por la ciudadanía hacia los gobernantes.

Tomando estos antecedentes teóricos como referencia, en el presente artículo se busca determinar en qué medida la dependencia del sistema mediático influyó en la evaluación realizada por la ciudadanía mexicana de la gestión gubernamental ante la afectación por la pandemia de Covid-19 en el país. Además, se busca determinar si factores de índole personal, como puede ser el partidismo manifestado por los ciudadanos, tienen la capacidad de moderar el efecto antes señalado. Para ello, se tomará como caso de estudio 
la evaluación de la gestión de los dos principales actores involucrados a nivel federal en México en la gestión de la pandemia. Por una parte, el presidente de la república Andrés Manuel López Obrador, en tanto que es el representante del Poder Ejecutivo. Por la otra, se estudiará la evaluación del subsecretario de Salud, ${ }^{2}$ Hugo López-Gatell Ramírez, quien ha sido el portavoz gubernamental en materia de salud, manteniendo una comunicación permanente con la ciudadanía a través de una conferencia de prensa diaria desde el 28 de febrero de 2020 y ocasionales a partir de mediados de junio de 2021.

\section{La dependencia del sistema mediático en situaciones de crisis}

Los medios de comunicación constituyen herramientas cruciales para el acceso de la ciudadanía a la información sobre la realidad social (Shehata y Strömbäck, 2014), una actividad que se ha extendido desde los medios más tradicionales, como la prensa, radio o televisión, hacia los más actuales, como pueden ser los medios y redes sociales. Esta relación tiende a hacerse más robusta en la medida que la población requiera de una mayor orientación para tomar decisiones, algo que puede suceder en situaciones de cambio e incertidumbre, como ante una guerra, un atentado terrorista, una crisis de salud o en un contexto de mayor normalidad como puede ser una campaña electoral (Mehrad y Yousefi, 2018; Morton y Duck, 2001; Muñiz, 2011). Es habitual que en estos contextos de incertidumbre la dependencia de la información transmitida por los medios de comunicación se incremente, haciéndose las personas más dependientes de los mensajes recibidos a través de estas vías de comunicación (Halpern, 1994; Hindman, 2004; Tai y Sun, 2007). Algo que ya se ha detectado en diferentes países respecto de la pandemia de Covid-19, donde ha aumentado el consumo mediático para obtener información relativa a la contingencia, cada vez más a través de la información recibida no solamente por los medios tradicionales, sino por las vías informativas creadas por los medios digitales (Casero-Ripollés, 2020; Duc Huynh, 2020).

Esta dependencia ha sido conceptualizada como aquella relación que se establece entre diferentes actores, en la cual la capacidad que unos tendrán de consumar sus objetivos dependerá del acceso a los recursos informativos

2 Si bien su cargo oficial es de subsecretario de Prevención y Promoción de la Salud dentro de la Secretaría de Salud del Gobierno de México, a efectos de este artículo se utilizará la denominación de subsecretario de Salud, que es la habitualmente empleada en los medios de comunicación. 
que ostentan otros (Ognyanova y Ball-Rokeach, 2015). Esta fuerte dependencia que se genera entre públicos y medios de comunicación en momentos de cambio y conflicto, como pueden ser las crisis, ha sido explicada desde la teoría de la dependencia del sistema mediático (Ball-Rokeach y Defleur, 1976; Shehata y Strömbäck, 2014). El postulado principal de la teoría mantiene que los medios se convierten en fuentes informativas primarias en momentos conflictivos dentro del sistema social, ya sean resultado de desastres naturales o debido a la mano humana (Ball-Rokeach y Defleur, 1976; Hindman, 2004). Esta acción de los medios se enmarca desde la teoría en la interrelación que existe entre medios, sociedad y audiencia, la cual permite además comprender el uso mediático de los individuos en situaciones de crisis (Hindman, 2004; Tai y Sun, 2007).

La relación de dependencia puede plasmarse en un nivel macro, donde se presentan relaciones entre el sistema mediático y el sistema político; pero también a nivel micro, donde se hacen manifiestos los efectos del sistema mediático sobre la población (DeFleur y Ball-Rokeach, 1989). Se observa, por tanto, que la teoría se sitúa a medio camino entre aquellas más centradas en el estudio de los efectos derivados del consumo de contenidos mediáticos, como puede ser la teoría del establecimiento de la agenda o del framing, y las que se centran en estudiar los hábitos y expectativas mantenidas por la audiencia para consumir medios, aspecto abordado desde la teoría de usos y gratificaciones (Mehrad y Yousefi, 2018). Así, se asume que las personas se exponen a los contenidos mediáticos para satisfacer ciertas necesidades psicológicas, incluidas las de orientación, comprensión y actuación (Hindman, 2004; Lin et al., 2020). En particular, se ha detectado que la dependencia individual del sistema mediático constituye una orientación previa que lleva a las personas a buscar información para comprender el ambiente social, obtener guías de cara a relacionarse con otras personas e incluso actuar ante los problemas siguiendo las normas sociales establecidas (Ball-Rokeach y Defleur, 1976; Davies, 2009; Mehrad y Yousefi, 2018; Wilkin y Ball-Rokeach, 2006).

Por tanto, se puede asumir que la atención mediática de las personas será más fuerte en la medida en que se incremente su dependencia del sistema mediático (Halpern, 1994; Tai y Sun, 2007). Algo que tiende a enfatizarse en contextos de cambio y conflicto, como la crisis suscitada por la pandemia de Covid-19, donde es habitual que la población tienda a acrecentar sus niveles de búsqueda de información sobre temas de salud ofrecida por fuentes tanto oficiales como no oficiales, así como a través de los medios más tradicionales y también de las nuevas vías de información proporcionadas por los medios 
digitales (So et al., 2019; Yoo et al., 2020). Ahora bien, son diferentes los factores que pueden influir en el hecho de que esta búsqueda de información se acreciente, entre los que se pueden citar tanto los contextuales como los individuales. Entre estos últimos se han detectado como principales el desarrollo de emociones de miedo o ansiedad y la percepción de riesgo generadas en las personas ante los posibles efectos negativos que a nivel individual pueden derivar de la crisis que se esté viviendo (So et al., 2019).

Este incremento del procesamiento de las noticias e informaciones transmitidas por los medios derivado de la relación de dependencia genera, de forma complementaria, un aumento de la probabilidad de que esos medios impacten el desarrollo de creencias, percepciones, sentimientos y comportamientos de la población mediante la diseminación de la información realizada en sus noticias (Ball-Rokeach y Defleur, 1976; Halpern, 1994; Hindman, 2004; Morton y Duck, 2001; Ognyanova y Ball-Rokeach, 2015). Ello se debe a que los medios permiten la obtención de información confiable para tomar decisiones, como el establecimiento de comportamientos de protección ante la crisis de salud particular (Garfin et al., 2020; So et al., 2019). Así, se ha señalado que la dependencia puede generar en las personas un mayor conocimiento sobre las crisis (Lin et al., 2020) o la realización de ciertos comportamientos, como pueden ser acciones de prevención promovidas por las autoridades gubernamentales y de salud (Morton y Duck, 2001; Yoo et al., 2020).

Además, se han detectado efectos actitudinales de la dependencia, como el incremento durante las crisis de emociones de miedo o ansiedad (Mehrad y Yousefi, 2018) o de la percepción de riesgo personal (Morton y Duck, 2001; Muñiz, 2011; Yoo et al., 2020). Pero también sobre las percepciones y comportamientos políticos (Halpern, 1994), debido a la fuerte dependencia que tienen los ciudadanos del sistema mediático para obtener información, por ejemplo, sobre los candidatos en una campaña electoral o sobre las decisiones políticas de gobernantes y legisladores en su gestión diaria (Davies, 2009; Shehata y Strömbäck, 2014). Hasta el momento, los estudios han observado que la dependencia es un claro predictor de las percepciones políticas ciudadanas (Halpern, 1994), las decisiones de voto (Davies, 2009) o la eficacia política (Ognyanova y Ball-Rokeach, 2015). Por ejemplo, Hindman (2004) detectó que el apoyo del presidente en Estados Unidos tendió a aumentar entre quienes tenían más dependencia mediática durante la crisis generada por el ataque terrorista del 11-S de 2001. 


\section{Evaluación de la gestión política durante las crisis}

Los gobernantes buscan resolver con su gestión de la política diaria los problemas que afectan a la sociedad a la que representan. Pero más allá de esta labor de gestión pública, no cabe duda de que sus acciones también vienen determinadas por una estrategia de comunicación con la cual se busca impactar a la opinión pública, para determinar la imagen hacia el gobierno y sus representantes. No en vano, como mencionan Krosnick y Kinder (1990: 497), la popularidad es "un recurso político vital" que todo gobernante siempre trata de cuidar. En gran medida, la gestión política que los gobernantes hagan de los asuntos que afectan socialmente determinará la evaluación que los ciudadanos hagan de esos gestores, es decir, la aprobación que se haga de su desempeño político (Donovan et al., 2020). Por ello, esta evaluación ciudadana, reflejada a través de los diferentes estudios de opinión que se desarrollan habitualmente, es un factor deseado, buscado y supervisado de forma permanente por las élites políticas (Dempsey, 2006).

Una realidad que se presenta especialmente durante momentos de crisis o ante sucesos imprevistos, los cuales, en palabras de Canel (2009: 220), constituyen cualquier tipo de "evento o suceso que escapa a los planes o programación política que tiene el gobierno”, como por ejemplo: atentados terroristas, guerras, crisis económicas o crisis de salud. Así, se ha señalado que la gestión, más o menos acertada, que hagan los gobernantes de los sucesos imprevistos con los que se encuentren en su administración tiene un fuerte impacto en la valoración y popularidad de la que gozará el Ejecutivo entre los ciudadanos (Hindman, 2004; Krosnick y Brannon, 1993). Este efecto se presenta con especial fuerza respecto del presidente - bien de la república en un sistema presidencialista o del gobierno en uno parlamentario - , al constituirse en el líder del Poder Ejecutivo y personificar a la propia institución (Canel, 2009; Krosnick y Kinder, 1990).

En este sentido, se ha demostrado que la gestión gubernamental para superar o lidiar con determinadas crisis deriva en cambios en su popularidad. En el caso de Estados Unidos, Krosnick y Brannon (1993) detectaron cómo la gestión de la Guerra del Golfo durante 1991 tuvo un impacto importante en la visión de la actuación presidencial por parte de la ciudadanía. Lo mismo sucedió respecto a la actuación de los diferentes gobernantes ante los ataques terroristas del 11 de septiembre de 2001. Así, la popularidad del alcalde de Nueva York (Canel, 2009) y del presidente del país (Hindman, 2004) aumentaron tras el evento. En el caso español, se ha comprobado que la gestión realizada de diversos eventos imprevistos acaecidos durante gobiernos 
de diferente signo político tuvo una influencia directa en la popularidad y valoración de la gestión del gobierno, lo cual refleja que los eventos funcionan como estimuladores del apoyo público (Canel, 2009).

Ahora bien, para que la gestión tenga un peso importante en la evaluación del gobernante, es necesario primero que ésta sea conocida por la opinión pública. En este sentido, aunque algunos autores ven a los medios como causas no relevantes de la interpretación pública de las acciones gubernamentales (Dempsey, 2006), tradicionalmente se asume que los medios constituyen herramientas esenciales para dar a conocer la gestión y que, por tanto, inciden en la evaluación ciudadana a través de la atención que hagan de ella (Todhunter, 2013). Esta relación entre el sistema político y el mediático constituye uno de los procesos básicos dentro del nivel macro de la dependencia del sistema mediático, que explica cómo los medios se vuelven altamente dependientes de las élites políticas y gobernantes para elaborar sus noticias sobre las crisis (Hindman, 2004; Ognyanova y Ball-Rokeach, 2015). Por ello, no es de extrañar que en contextos de crisis y fuerte incertidumbre los gobiernos traten de controlar la comunicación que se hace hacia la sociedad para su beneficio político (Canel, 2009).

Ello se debe a que es habitual que los medios de comunicación establezcan "los estándares a través de los cuales los gobernantes, presidentes, cargos públicos y candidatos políticos son juzgados” (Iyengar y Kinder, 1987: 63). Así, las evaluaciones suelen estar condicionadas sustancialmente por la cobertura de las noticias a las acciones de los gobernantes ante asuntos relevantes, como puede ser una crisis (Donovan et al., 2020; Krosnick y Kinder, 1990; Sheafer y Weimann, 2005). En este sentido, cuanta más atención presta el medio a un asunto, más fuertemente se preactiva éste en la mente del público y más probable es que se haga accesible para evaluar al gobierno (Krosnick y Kinder, 1990; Scheufele, 2000). Al respecto, se ha detectado que en tiempos de alta dependencia del sistema mediático, el consumo mediático puede impactar en el apoyo gubernamental, como ocurrió tras el ataque terrorista del 11-S en Estados Unidos (Hindman, 2004).

Sin embargo, esta exposición no es uniforme entre todos los integrantes de la audiencia, en tanto que la dependencia a los diferentes medios, tanto tradicionales como sociales, estriba en las características personales de cada individuo (Lin et al., 2020; Mehrad y Yousefi, 2018). Al respecto, se ha apuntado que hay diferentes factores de índole personal que pueden moderar el efecto de la dependencia del sistema mediático (Ball-Rokeach, 1998; Mehrad y Yousefi, 2018). Aspectos como el sexo, la edad o el nivel educativo de las personas tienen la capacidad de moderar la influencia de la exposición 
en la valoración de las acciones de gobierno ante una crisis (Mehrad y Yousefi, 2018). Junto a ellos, el partidismo ha sido detectado habitualmente como un elemento moderador clave del efecto que tiene el consumo mediático sobre la evaluación que la opinión pública realiza del desempeño gubernamental (Hindman, 2004). En gran medida, debido a que este público tiende a contar con mayor experiencia o involucramiento en política, lo cual de forma habitual le lleva a estar más informado (Krosnick y Brannon, 1993).

Aunque no es abundante la investigación al respecto, este efecto moderador ya se ha detectado en situaciones como el apoyo público de la gestión del presidente de Estados Unidos ante los ataques terroristas del 11-S de 2001. En su trabajo, Hindman (2004) detectó que el partidismo hizo que la aprobación fluctuara en función de la cercanía ideológica del encuestado. Este resultado permitió al autor descartar un posible efecto rally round the flag, que plantea cómo ante situaciones de crisis los predictores que tradicionalmente determinan la aprobación presidencial pueden volverse menos predictivos de lo que usualmente son durante la gestión cotidiana de los gobiernos. Así, las preferencias ideológicas o partidistas mantenidas por la ciudadanía pueden ser desplazadas por una tendencia de apoyo y aprobación gubernamental en todos los sectores como reacción a la crisis (Hindman, 2004; Hetherington y Nelson, 2003; McLeod et al., 1994). Un efecto que, en todo caso, cabe esperar que decaiga conforme transcurra el tiempo y los factores ideológicos o partidistas vuelvan a ser centrales en el debate político acerca de la gestión gubernamental y de sus resultados (Gartner y Segura, 1998). Teniendo en cuenta los antecedentes teóricos aportados, se plantean las siguientes hipótesis y preguntas de investigación en este estudio:

PI1: ¿Existen diferencias en la evaluación de la gestión realizada durante la pandemia por parte del presidente y del subsecretario de Salud?

H1: La dependencia del sistema mediático contribuye a aumentar la evaluación de la gestión realizada por el presidente y el subsecretario de Salud.

$\mathrm{H} 2$ : El partidismo tiene un efecto moderador sobre la relación entre la dependencia del sistema mediático y la evaluación de la gestión de la pandemia realizada por parte del presidente y del subsecretario de Salud. 


\section{Método seguido}

\section{Diseño y muestra}

Se efectuó una encuesta descriptiva a nivel nacional mexicano, a través de la aplicación de un cuestionario a una muestra probabilística de la población. Teniendo en cuenta la situación causada por la pandemia, que recomendó no realizar trabajo de campo en vivienda, se decidió contratar a la empresa QuestionPro que está especializada en el levantamiento de encuestas mediante entrevistas en línea a nivel internacional. Al respecto, cabe señalar que aunque el levantamiento de campo se hizo en línea, el ejercicio demoscópico que se presenta en este artículo no constituye una encuesta en línea tradicional. En este sentido, la selección de la muestra para la encuesta fue realizada de entre los integrantes del panel o grupo nacional de participantes (pool of respondents) en los estudios de la empresa, mediante una invitación personal enviada únicamente a aquellos integrantes de dicho panel o grupo nacional que presentaran las características que fueron requeridas, para garantizar una adecuada representatividad final de la muestra.

En todo caso, es posible que la realización de entrevistas en línea para completar una encuesta en la actualidad en México pueda representar cierto sesgo, que viene en gran medida determinado por el nivel de penetración que internet tiene en el país. Al respecto, los datos actuales reflejan una cada vez mayor disminución de la brecha digital para la población general. Así lo atestiguan datos como los aportados por el Instituto Nacional de Estadística y Geografía (INEGI, 2020), los cuales ponían de manifiesto que para 2020 aproximadamente $78 \%$ de la población mexicana tenía acceso a internet, lo que representaba cerca de 84 millones de usuarios. Estos datos son confirmados con otros más recientes (World Internet Stats, 2021), que estiman en torno a 92 millones el número de internautas existentes en el país (71\% de la población), aumentando hasta 100 millones los usuarios de redes sociales, lo cual equivalía a $77.2 \%$ de la población.

Tomando esto como referencia, los datos obtenidos a través de la encuesta realizada para este estudio permiten tener una visión acerca de la percepción y afectación que supuso la pandemia por Covid-19 entre la población mexicana. El trabajo de campo y levantamiento de datos se llevó a cabo entre el 1 y el 8 de septiembre de 2020. La recolección de datos se realizó mediante un cuestionario diseñado en la plataforma QuestionPro, que da la posibilidad de impedir que el mismo participante conteste más de una vez la encuesta, para evitar así el denominado "relleno de urnas" (ballot box stuffing). 
Ello permite reducir, además, posibles sesgos derivados de la aplicación de entrevistas en línea. Se buscó contar con una muestra representativa de la población mexicana en términos de sexo, edad y zonas de residencia. Para ello se llevó a cabo, en primer lugar, un muestreo probabilístico estratificado, utilizando como marco muestral las zonas Nielsen de México, que permiten estratificar al país en seis zonas geográficas, a partir de las cuales se trabajó para obtener una muestra por afijación proporcional.

Además, a nivel nacional se estratificó con afijación proporcional por las variables de sexo (hombre vs. mujer), edad (18-29 años, 30-39 años, 40-49 años, 50-59 años y 60 años en adelante) y el nivel socioeconómico, medido mediante el índice de Nivel Socioeconómico (NSE) desarrollado por la Asociación Mexicana de Agencias de Inteligencia de Mercado y Opinión (AMAI, 2017). Tomando en consideración estas características, se seleccionó la muestra del estudio a partir del panel de la empresa, procediendo a realizar invitaciones individuales entre sus integrantes hasta completar cada uno de los estratos establecidos. En total participaron en la encuesta 1,211 adultos mayores de edad, con una tasa de rechazo a las entrevistas de $18 \%$ y una duración promedio de respuesta de 21 minutos. La muestra trabajó con un margen de error de $+/-2.8 \%$ y con un nivel de confianza de $95 \%$.

\section{Medidas utilizadas}

Dependencia del sistema mediático: En relación con este constructo, se tomó como referencia la adaptación de Muñiz (2011) para el caso mexicano del instrumento de Morton y Duck (2001), que mide el grado de utilidad que dieron los encuestados a los medios de comunicación para conocer y comprender la información sobre la pandemia. En concreto, se evaluó el grado de acuerdo con las siguientes afirmaciones: "Nos mantienen informados sobre temas de salud importantes, como el coronavirus", "Ayudan a comprender la importancia que temas como el coronavirus tienen para la salud", "Permiten decidir qué precauciones de salud tomar ante situaciones como el coronavirus" y "Posibilitan conocer cómo otras personas hacen frente a temas importantes de salud, como el coronavirus". Todas las preguntas se midieron a través de una escala tipo Likert con cinco puntos de respuesta: desde nada (1), hasta mucho (5). La consistencia interna del indicador fue alta $(\alpha=.88)$, por lo que se procedió a generar un índice promediando los cuatro reactivos antes mencionados. 
Evaluación a la gestión de los gobernantes: Con el objetivo de determinar la evaluación que hacían los participantes de la gestión respecto a la pandemia por coronavirus realizada por los gobernantes, se solicitó que se indicara cómo valoraban la actuación de una serie de actores políticos involucrados con la pandemia en México y a nivel mundial. Para ello, se utilizó una escala que oscilaba entre muy mala (0) y muy buena (10), pasando por una evaluación regular (5). Se incluyó un listado de seis actores, con el propósito de no desviar la atención de los participantes hacia los dos actores de los que se buscaba en particular determinar la evaluación de su gestión. En concreto, se trabajó con los datos relativos al presidente Andrés Manuel López Obrador y al subsecretario de Salud Hugo López-Gatell Ramírez, al ser los dos actores con mayor saliencia mediática a nivel federal durante la pandemia a nivel nacional.

Partidismo: Se pidió a los participantes en la encuesta que indicaran a qué partido político de los existentes a nivel nacional mexicano se sentían más cercanos. De esta manera, se podía señalar al Partido Acción Nacional (PAN), al Partido Revolucionario Institucional (PRI), al Partido de la Revolución Democrática (PRD), al Partido Verde Ecologista de México (PVEM), al Partido del Trabajo (PT), al Movimiento Regeneración Nacional (Morena), a Movimiento Ciudadano, al Partido Nueva Alianza (PANAL) o a otro partido. Asimismo, se presentó la opción "Ninguno" para aquellos ciudadanos no cercanos a partidos políticos. De cara al análisis estadístico, todos los cercanos a partidos se concentraron en una categoría de partidistas (2), mientras que los restantes constituyeron la categoría de no partidistas (1).

Variables de control: Finalmente, se contempló el uso de una serie de variables de control de carácter sociodemográfico. En concreto, se les cuestionó a los participantes acerca de su género $(1=$ masculino; $2=$ femenino $)$ y su edad, en años cumplidos. También se midió el nivel socioeconómico a partir del índice NSE creado por la AMAI (2017). La escala está compuesta por siete niveles, que oscilan desde el nivel alto (A/B) hasta el nivel más bajo (E), donde los niveles socioeconómicos vinculados con la clase media estarían entre los niveles: C+, C y C-. También se evaluó el nivel de estudios de los participantes, con una escala que osciló entre: no tiene (1) y estudios de posgrado (7).

\section{Análisis de los resultados}

En respuesta a la primera pregunta de investigación, se observan diferencias estadísticamente significativas en la evaluación hecha por parte 
de la ciudadanía respecto a la gestión de la pandemia realizada por ambos actores políticos, $F(1,1206)=6.103, p=.014, \eta_{\text {parcial }}^{2}=.005$. Si bien las diferencias fueron pequeñas, atendiendo al tamaño del efecto encontrado, se pudo determinar que la puntuación del presidente $(M=4.98$, $D E=3.63)$ fue sensiblemente menor que la del subsecretario de salud $(M=5.76, D E=3.34)$. Esta diferencia se acrecienta de forma sensible al comparar la puntuación modal de ambos actores detectada en la encuesta, pues mientras que el presidente presentaba una puntuación modal de 0 puntos, la del subsecretario de Salud se situaba en 5 puntos (véase Figura $1^{3}$ ).

Por otra parte, y atendiendo a lo planteado en la primera hipótesis, se procedió a calcular las relaciones existentes entre las variables del estudio, con el objetivo de determinar en qué medida había relaciones estadísticamente significativas entre la dependencia del sistema mediático mantenida por los encuestados durante la pandemia con la evaluación que hacían de la gestión desarrollada por los actores políticos de la crisis. Para ello, se realizaron regresiones lineales múltiples jerárquicas para explicar la evaluación de cada actor político. Con esta técnica -que permite introducir las variables independientes en la ecuación en diferentes momentos- es posible generar modelos explicativos distintos y detectar las variaciones producidas en el efecto por las diferentes variables predictoras sobre las variables criterio analizadas. En ambos casos, dentro del primer bloque se incorporaron a la ecuación las variables sociodemográficas contempladas en el estudio (modelo 1) con el fin de controlar su posible impacto, debido a que constituyen potenciales variables moderadoras del modelo. Con ello se buscó poder evidenciar el impacto moderador de la cercanía partidista, una vez eliminada la covariación o influencia de otras posibles variables que se han detectado en la literatura como moderadoras (Ball-Rokeach, 1998; Mehrad y Yousefi, 2018). Inmediatamente después se incorporó la dependencia del sistema mediático (modelo 2) y el partidismo (modelo 3 ) dentro de la ecuación para determinar su impacto.

En cuanto al análisis del impacto sobre la evaluación de la gestión de la pandemia realizada por el presidente (véase Tabla 1), las variables utilizadas en la regresión cumplieron el supuesto de independencia respecto de la variable dependiente, atendiendo a que el valor del test de Durbin-Watson fue aceptable $(d=2.03)$ y por tanto superior a los límites inferior de $1.5 \mathrm{y}$ superior de 2.5 aceptados. Además, se descartaron problemas de colinealidad

3 Todas las figuras y las tablas se encuentran en el Anexo, al final del presente artículo (Nota del editor). 
entre las variables usadas en cada ecuación, resultando el modelo estadísticamente significativo, $F(6,1204)=25.874, p<.001, R^{2}=.114$. Entrando a los resultados, el primer modelo logró explicar $1.5 \%$ de la evaluación de la gestión presidencial, constituyendo el sexo $(\beta=-.089, p=.002)$ y el nivel educativo $(\beta=-.077, p=.009)$ las variables con impacto estadísticamente significativo. La inclusión de la dependencia del sistema mediático en la ecuación hizo aumentar hasta 3.3\% la explicación de la evaluación $(\beta=.136, p<.001)$, mientras que la inclusión del partidismo explicó $8.1 \%$ de la evaluación presidencial $(\beta=.290, p<.001)$. Por lo tanto, se observa que la dependencia influyó positivamente en la evaluación de la gestión del presidente, una relación que se vio moderada por la inclusión en la ecuación del partidismo que aunque atenuó el anterior impacto, no llegó a suprimirlo.

En cuanto al análisis del impacto sobre la evaluación de la gestión de la pandemia realizada por el subsecretario de salud (véase Tabla 2), las variables utilizadas en la regresión también cumplieron el supuesto de independencia respecto de la variable dependiente, atendiendo a que el valor del test de Durbin-Watson fue aceptable $(d=1.96)$. Además, se descartaron problemas de colinealidad entre las variables usadas en cada ecuación, resultando el modelo estadísticamente significativo, $F(6,1204)=16.557, p<.001$, $R^{2}=.076$. Al revisar los resultados, se observa que el primer modelo logró explicar $1.6 \%$ de la evaluación de la gestión presidencial, constituyendo en este caso la edad $(\beta=-.124, p<.001)$ la única variable sociodemográfica con un impacto estadísticamente significativo. La inclusión de la dependencia del sistema mediático en la ecuación hizo aumentar hasta $4.2 \%$ la explicación de la evaluación $(\beta=.207, p<.001)$, mientras que la inclusión del partidismo explicó $1.9 \%$ de la evaluación presidencial $(\beta=.139, p<.001)$. De nuevo, la dependencia influyó positivamente en la evaluación de la gestión del actor, atenuando el partidismo este impacto pero sin llegar a suprimirlo.

A pesar de los análisis efectuados, estos resultados no permiten aclarar totalmente el posible impacto de moderación de la variable de partidismo, ni tampoco si existen diferencias entre los distintos niveles de partidismo. Por ello, y tomando como referencia la segunda hipótesis de investigación, se realizó un análisis mediacional para determinar si el partidismo moderaba la influencia de la dependencia sobre la evaluación de la gestión de la pandemia. Para ello, se utilizó la macro PROCESS para SPSS que permite calcular el efecto principal de la variable independiente o predictora sobre la variable dependiente o criterio (efecto b1), el efecto principal de la variable moderadora sobre la variable dependiente (efecto b2) y el efecto de interacción de las variables independiente y moderadora sobre la variable dependiente (efectos 
b3) (Hayes, 2013). En concreto, se usó el modelo 1, con un bootstrapping de 10,000 muestras, para calcular los tres efectos antes descritos, mismo que se apoya en un análisis de regresión lineal múltiple efectuado con las variables contempladas en el estudio.

Esta técnica ha sido vista como la más adecuada cuando se busca determinar el efecto de interacción entre una variable continua -en este caso la variable correspondiente al nivel de dependencia del sistema mediático-y una variable dicotómica -en este caso correspondiente a la de partidismo-. Como paso previo a la realización del estudio, la variable de partidismo fue convertida en una variable dummy, donde la categoría no partidista fue codificada con un valor de -0.05 y la categoría partidista con un valor de 0.05 . Se ha señalado que este modelo es el más adecuado, pues permite delimitar las diferentes "regiones de significatividad estadística", a partir del uso de la técnica Johnson-Neyman; es decir, posibilita observar el efecto de la variable independiente sobre la dependiente en los distintos valores adoptados por la variable moderadora.

En primer lugar, se calculó el efecto de moderación del partidismo en la evaluación de la gestión del presidente (véase Figura 2). Los resultados mostraron que el modelo era estadísticamente significativo, $F(7,1203)=22.534, p<.001$, y que en conjunto explicaba $11.59 \%$ de la varianza. Por una parte, se detectó un efecto principal de la dependencia sobre la evaluación, $B_{\text {dependencia }}=.46, E T=.13, p<.001$, 95\% IC [.2098, .7097], lo cual pone de manifiesto que a mayor dependencia del sistema mediático, mayor era la evaluación de la gestión de la pandemia realizada por el presidente. Por otra parte, no se observó un efecto principal del partidismo, $B_{\text {partidismo }}=.60, E T=1.02, p=.558,95 \%$ IC $[-1.4105$, 2.6101], pero sí un efecto moderador del partidismo en la influencia de la dependencia. En particular, este efecto de interacción se presentó en la categoría de partidistas, $B=.65, p<.001, E T=.16, p<.001,95 \%$ IC [.3379, .9666], entre quienes una mayor dependencia del sistema mediático contribuía a aumentar sus niveles de apoyo a la gestión del presidente durante la pandemia. Sin embargo, entre los no partidistas no se observó este efecto de moderación de la variable, $B=.27, p<.001, E T=.20, p=.175,95 \%$ IC $[-.1189, .6533]$, manteniéndose por tanto en este grupo la evaluación sin alteraciones observables.

En segundo lugar, se calculó el efecto de moderación del partidismo en la evaluación de la gestión del subsecretario de Salud (véase Figura 3). Los resultados mostraron que el modelo era estadísticamente significativo, $F(7,1203)=14.223$, $p<.001$, y que en conjunto explicaba $7.65 \%$ de la varianza. Por una parte, se detectó un efecto principal de 
la dependencia del sistema mediático sobre la evaluación de la gestión del subsecretario, $B_{\text {dependencia }}=.80, E T=.12, p<.001,95 \%$ IC $[.5640,1.0335]$. Sin embargo, no se observó un efecto principal del partidismo, $B_{\text {partidismo }}=.42, E T=.96, p=.664,95 \%$ IC $[-1.4707,2.3056]$. Frente a este resultado, sí se detectó la existencia de un efecto significativo de moderación del partidismo sobre la influencia ejercida por la dependencia. En particular, este efecto de interacción se presentó tanto en la categoría de no partidistas, $B=.73, E T=.18, p<.001,95 \%$ IC $[.3707,1.0960]$, como de los partidistas, $B=.86, E T=.15, p<.001,95 \%$ IC $[.5689,1.1594]$. En este sentido, en ambos grupos una mayor dependencia del sistema mediático contribuía a generar un incremento en sus niveles de apoyo a la gestión realizada por el subsecretario de Salud durante la pandemia.

\section{Discusión y conclusiones}

Este artículo se planteó como objetivo determinar hasta qué punto el nivel de dependencia mediática mantenida por los ciudadanos durante la pandemia de Covid-19 en México influyó en la evaluación de la gestión llevada a cabo por los dos actores clave en la crisis en el país: el presidente de la república Andrés Manuel López Obrador y el subsecretario de Salud Hugo LópezGatell Ramírez. Junto a ello, el estudio buscaba determinar si el partidismo manifestado por los ciudadanos - un factor de índole personal que les diferencia de los ciudadanos declarados como no partidistas- tuvo la capacidad de influir sobre la relación antes señalada. Es decir, se buscó determinar si el partidismo tuvo algún tipo de influencia moderadora en la relación entre la dependencia mediática y la evaluación de los dos gobernantes a nivel federal en México mantenida por los participantes. Para ello, el estudio se apoyó en los datos de una encuesta representativa a nivel nacional, con la cual se buscaba dar respuesta a la pregunta de investigación y contrastar las hipótesis planteadas.

En primer lugar, y en relación con la pregunta de investigación que cuestionaba si existieron diferencias en la evaluación de la gestión realizada por ambos gobernantes, los datos obtenidos permiten dar una respuesta al respecto. En este sentido, se observa cómo a nivel estadístico ambas valoraciones difirieron, aunque cabe reconocer que las diferencias fueron pequeñas atendiendo al tamaño del efecto. Junto a este análisis cuantitativo, una revisión más cualitativa de los datos permite reafirmar esta conclusión, pues mientras el presidente aglutinó una fuerte evaluación negativa expresada en una puntuación modal de 0 , en torno a la persona del subsecretario se 
agrupó una evaluación más positiva al presentar una puntuación modal de 5. En todo caso, este resultado pone de manifiesto la necesidad de mejorar los instrumentos de medición utilizados habitualmente para determinar el grado de apoyo o aprobación de la gestión de los gobernantes, para hacerlos más precisos y sensibles ante las diferencias sutiles con carga emocional que cada vez parecen estar más presentes entre la ciudadanía.

En relación con la primera hipótesis de investigación, los hallazgos permiten observar cómo la dependencia del sistema mediático determina en buena medida la evaluación pública de la gestión de los gobernantes. En este sentido, y en consonancia con la hipótesis planteada, se detectó que la dependencia es un claro predictor de la evaluación que los ciudadanos mantienen acerca de la gestión realizada por los gobernantes en contextos de conflicto o crisis, como fue la pandemia por Covid-19. Además, se observa que esta relación se presenta para ambos gobernantes en términos similares a los detectados previamente por Hindman (2004), en su estudio sobre el apoyo público al presidente de Estados Unidos en su gestión ante la crisis provocada por el ataque terrorista del 11-S. Cabe mencionar también que este resultado se observa tanto para el presidente como para el subsecretario de Salud, a pesar de que la evaluación de ambos actores fuera estadísticamente significativa. Estos resultados contribuyen a profundizar el debate acerca de los efectos políticos de la dependencia del sistema mediático, pues aunque esta relación parece clara, son pocos los estudios que la han abordado empíricamente hasta la fecha (Davies, 2009; Halpern, 1994; Hindman, 2004; Ognyanova y BallRokeach, 2015).

Por otra parte, la segunda hipótesis planteada en el artículo se confirmó plenamente, al poderse detectar el efecto de moderación desarrollado por el partidismo en la relación de la dependencia del sistema mediático y con la evaluación de la gestión llevada a cabo por ambos actores políticos durante la crisis, en línea con lo encontrado por Hindman (2004) en un contexto geográfico diferente. Se concluye, por tanto, que el hecho de sentirse cercano o no a los partidos políticos supuso un factor determinante para que la evaluación de la gestión gubernamental durante la pandemia se viera alterada por la dependencia del sistema mediático. A pesar de ello, es importante destacar que el efecto del partidismo fue diferente en relación con la evaluación de cada actor. En particular, se ha comprobado que entre los más cercanos a partidos -independientemente del partido al que se sintieran más vinculados-, su dependencia mediática tendía a hacer aumentar la evaluación de ambos actores. Sin embargo, entre los no partidistas las variaciones sólo se presentaron respecto a la evaluación del subsecretario de Salud, pero no así del presidente. 
Esto lleva a reflexionar cómo la cercanía partidista de los ciudadanos puede condicionar el efecto de la dependencia mediática sobre la evaluación de la gestión gubernamental. Es posible que ciertos factores contextuales puedan propiciar que el efecto del partidismo sea más pronunciado, como es el ambiente político que cada vez se manifiesta más en una fuerte polarización que viven en el presente las sociedades en el terreno ideológico (Donovan et al., 2020). La política mexicana no escapa de este escenario de polarización, que se manifiesta en una brecha cada vez más profunda en la opinión pública. Algo que se ha acrecentado tras la llegada de López Obrador a la presidencia en 2018, quien en su discurso político tiende a trabajar con una narrativa de ruptura entre "ellos" y "nosotros" (Moreno, 2019). Es posible que aquí radique el hecho de que sea entre los partidistas donde se produzca la mayor influencia de la dependencia mediática sobre la evaluación presidencial, pues son ellos los que se encuentran más involucrados en el debate político y en quienes el ambiente político puede tener una mayor capacidad de influencia.

Por otra parte, ante situaciones imprevistas, de cambio o incertidumbre como la pandemia de Covid-19, es habitual que buena parte de la ciudadanía presente un fuerte apoyo hacia los gobernantes, al margen de sus preferencias ideológicas o partidistas (Hetherington y Nelson, 2003; Hindman, 2004; McLeod et al., 1994). Este efecto rally round the flag lleva a desarrollar una evaluación positiva de aquellos gobernantes que son percibidos por la ciudadanía como gestores adecuados para atender la problemática presentada. Este puede haber sido el caso del subsecretario de Salud mexicano, teniendo en cuenta que su evaluación pública tendió a aumentar conforme mayor era la dependencia mediática entre la ciudadanía, independientemente de que existiera o no cercanía a partidos políticos. Estos datos llevan a reflexionar acerca del papel que los medios, a través de sus contenidos informativos, pudieron tener en estos efectos dispares para ambos gobernantes; ya que la configuración de la imagen de los gobernantes y la evaluación ciudadana sobre la gestión de éstos suelen estar condicionadas sustancialmente por la cobertura realizada por los medios (Donovan et al., 2020; Krosnick y Kinder, 1990).

Los resultados del presente estudio sugieren que, como se ha observado en otros contextos, en el caso mexicano también se pudo haber producido un efecto de preactivación de etiquetas o priming a partir de la cobertura realizada de los actores y su gestión de la pandemia de Covid-19 (Donovan et al., 2020; Krosnick y Brannon, 1993; Krosnick y Kinder, 1990). Así, frente a un rol más político del presidente e incluso contradictorio ante la crisis en la primera fase de la pandemia, es posible que el papel más técnico mantenido por el subsecretario de Salud le haya beneficiado ante la opinión 
pública, a partir de la imagen transmitida por los medios. En este sentido, el artículo abre nuevas vías para revisar las relaciones entre diferentes planteamientos teóricos que permitan estudiar y comprender la influencia que los medios tienen en las evaluaciones de los políticos y gobernantes. Por ejemplo, sería conveniente profundizar en el análisis del efecto priming o de preactivación jugado por los medios en contextos de alta dependencia mediática, a partir de la realización de estudios con acercamientos metodológicos con mayor control para determinar de forma precisa el impacto en actitudes y comportamientos políticos.

Finalmente, el estudio que configura el presente artículo ha permitido detectar ciertas limitaciones que se deberían solventar en futuras investigaciones, que puedan llevarse a cabo tomando como referencia los hallazgos de este trabajo. Sería recomendable que en futuros estudios también se abordara la acción comunicativa desarrollada por los gobiernos y sus representantes durante la gestión de la crisis, como la que ha supuesto la pandemia de Covid-19. No cabe duda de que, como recuerda Canel (2009), no es únicamente importante la gestión que hacen los gobiernos de las crisis, sino también la estrategia de comunicación que desarrollan para dar a conocer su gestión a la ciudadanía. Al respecto, se ha detectado que a menudo los gobernantes utilizan los desastres para construir una agenda política nueva que les beneficie, o incluso para solventar los problemas que arrastran en la agenda política existente (Hindman, 2004). Por ello sería muy recomendable abordar la construcción de esta agenda, así como del tratamiento de la realidad que trasladan a través de la promoción de ciertos encuadres (advocacy frames), con los cuales se busca impactar la interpretación periodística de la realidad política (Gerth y Siegert, 2012).

Además, cabe asumir que desde que la pandemia fuera declarada oficialmente en marzo de 2020, y los diferentes países (incluido México) tomaran las primeras decisiones para hacer frente a la contingencia, la situación vivida en torno a ésta ha evolucionado, pasando por diferentes fases y momentos. En este sentido, los resultados de la encuesta permiten determinar las percepciones ciudadanas acerca de la gestión gubernamental de la misma hasta el momento de su realización, circunscribiendo sus hallazgos a un momento y tiempo concreto dentro de la pandemia. Como ya se señaló en los antecedentes, en este tipo de crisis es habitual que los fuertes apoyos gubernamentales iniciales, o efecto rally round the flag, cedan con el tiempo a medida que la cercanía partidista vuelve a polarizar a la población (Hetherington y Nelson, 2003; Hindman, 2004; McLeod et al., 1994). Por eso cabe especular que de haberse efectuado el estudio en un momento inicial o posterior al de la 
encuesta, los resultados hubieran variado sensiblemente. En este sentido, la realización de investigaciones complementarias con datos recabados en otros momentos de la pandemia ayudaría a dar mayor fortaleza al modelo teórico estudiado y validado en este artículo.

Finalmente, también se observa una limitación metodológica relativa a la medición de la cercanía partidista realizada en la encuesta, que podría ser solventada de cara a efectuar futuros estudios que busquen probar con mayor precisión el efecto causal de la dependencia y cercanía partidista sobre la evaluación de la gestión gubernamental ante determinada crisis. La presente investigación, al tratarse de una encuesta de corte descriptivo, trabajó con una medición de la cercanía partidista mediante una variable categórica, lo cual dificulta modelar la cercanía a cada partido político como diferentes variables moderadoras del estudio. En todo caso, un contexto de polarización política como el que vive actualmente México aconsejaría hacer una futura investigación con un diseño metodológico que primara el alcance explicativo o causal frente al correlacional, para determinar de forma separada si la cercanía o identificación con el partido de gobierno y la cercanía al partido o partidos de oposición al presidente tienen distintos efectos mediadores en el modelo planteado. Se considera que llevar a cabo un análisis en este sentido ayudaría a incrementar la relevancia y aportaciones del trabajo para la literatura sobre el tema estudiado.

\section{Referencias}

AMAI (2017), Nivel Socio Económico AMAI 2018. Nota Metodológica, México: Asociación Mexicana de Agencias de Inteligencia de Mercado y Opinión (AMAI).

Ball-Rokeach, Sandra (1998), "A theory of media power and a theory of media use: Different stories, questions, and ways of thinking”, en Mass Communication \& Society, vol. 1, núm. 1-2, Inglaterra: Routledge.

Ball-Rokeach, Sandra y DeFleur, Melvin (1976), "A dependency model of mass-media effects”, en Communication Research, vol. 3, núm. 1, Estados Unidos: Sage.

Canel, María José (2009), "El impacto de los sucesos imprevistos en la imagen pública de los gobiernos españoles”, en Anàlisi: Quaderns de Comunicació i Cultura, núm. 38, España: Universitat Autònoma de Barcelona.

Casero-Ripollés, Andreu (2020), "Impact of Covid-19 on the media system. Communicative and democratic consequences of news consumption during the outbreak", en Profesional de la Información, vol. 29, núm. 2, España: Profesional de la Información.

Davies, John (2009), “The effect of media dependency on voting decisions”, en Journal of Media Sociology, vol. 1, núm. 3/4, Estados Unidos: Balios Books. 
DeFleur, Melvin y Ball-Rokeach, Sandra (1989), Theories of mass communication, Estados Unidos: Longman.

Dempsey, Jason (2006), "Public evaluation of presidential performance during foreign policy crises", en The Forum, vol. 4, núm. 1, Austria: De Gruyter.

Donovan, Kathleen et al. (2020), "Motivated reasoning, public opinion, and presidential approval”, en Political Behavior, vol. 42, Estados Unidos: Springer.

Duc Huynh, Toan Luu (2020), "The COVID-19 risk perception: A survey on socioeconomics and media attention”, en Economics Bulletin, vol. 40, núm. 1, Estados Unidos: Economics Bulletin.

Farré, Jordi (2005), “Comunicación de riesgo y espirales del miedo", en Comunicación y Sociedad, núm. 3, México: Universidad de Guadalajara.

Garfin, Dana Rose et al. (2020), "The novel coronavirus (COVID-2019) outbreak: amplification of public health consequences by media exposure", en Health Psychology, vol. 39, núm. 5, Estados Unidos: Kenneth E. Freedland.

Gartner, Scott Sigmund y Segura, Gary (1998), “War, casualties, and public opinion”, en Journal of Conflict Resolution, vol. 42, núm. 3, Estados Unidos: Sage.

Gerth, Matthias y Siegert, Gabriele (2012), "Patterns of consistence and constriction: How news media frame the coverage of direct democratic campaigns", en American Behavioral Scientist, vol. 56, núm. 3, Estados Unidos: Sage.

Halpern, Pablo (1994), "Media dependency and political perceptions in an authoritarian political system”, en Journal of Communication, vol. 44, núm. 4, Estados Unidos: Oxford University Press.

Hayes, Andrew (2013), Introduction to mediation, moderation, and conditional process analysis, Estados Unidos: Guilford.

Hetherington, Marc y Nelson, Michael (2003), "Anatomy of a rally effect: George W. Bush and the war on terrorism”, en PS. Political Science \& Politics, vol. 36, núm. 1, Estados Unidos: Cambridge University Press.

Hindman, Douglas (2004), "Media system dependency and public support for the press and president”, en Mass Communication and Society, vol. 7, núm. 1, Inglaterra: Routledge.

INEGI (2020), "Encuesta Nacional sobre Disponibilidad y Uso de Tecnologías de la Información en los Hogares (ENDUTIH) 2020”. Disponible en: https://www.inegi. org.mx/programas/dutih/2020/ [27 de septiembre de 2021].

Iyengar, Shanto y Kinder, Donald (1987), News that matters. Agenda setting and priming in a television age, Estados Unidos: University of Chicago Press.

Krosnick, Jon y Kinder, Donald (1990), "Altering the foundations of support for the president through priming”, en American Political Science Review, vol. 84, núm. 2, Estados Unidos: Cambridge University Press.

Krosnick, Jon y Brannon, Laura (1993), "The media and the foundations of presidential support: George Bush and the Persian Gulf conflict”, en Journal of Social Issues, vol. 49, núm. 4, Estados Unidos: Wiley.

Lin, Carolyn et al. (2020), "Information source dependence, presumed media influence, risk knowledge, and vaccination intention", en Atlantic Journal of Communication, Inglaterra: Routledge.

McLeod, Douglas et al. (1994), "Conflict and public opinion: Rallying effects of the Persian Gulf War”, en Journalism Quarterly, vol. 71, núm. 1, Estados Unidos: Sage. 
Mehrad, Jafary Yousefi, Zahra (2018), "Introducing the theory of'media system dependency' with emphasis on its potential application in theoretical framework of researches in the field of information science and knowledge", en International Journal of Information Science and Management, vol. 16, núm. 1, Irán: Journal of Information Science and Management (IJISM).

Moreno, Alejandro (2019), "López Obrador y la movilización del sentimiento popular", en Moreno, Alejandro, Uribe, Alexandra y Wals, Sergio [coords.], El viraje electoral: opinión pública y voto en las elecciones de 2018, México: Centro de Estudios Sociales y de Opinión Pública.

Morton, Thomas y Duck, Julie (2001), "Communication and health beliefs. Mass and interpersonal influences on perceptions of risk to self and others", en Communication Research, vol. 28, núm. 5, Estados Unidos: Sage.

Muñiz, Carlos (2011), "Búsqueda de información durante tiempos de crisis. Efectos de la comunicación interpersonal y masiva en la percepción de riesgo personal ante la gripe AH1N1", en Revista de Ciencias Sociales, vol. 17, núm. 1, Venezuela: Universidad del Zulia.

Ognyanova, Katherine y Ball-Rokeach, Sandra (2015), "Political efficacy on the internet: a media system dependency approach", en Communication and Information Technologies Annual, núm. 9, Inglaterra: Emerald.

Scheufele, Dietram (2000), "Agenda-Setting, priming and framing revisited: another look at cognitive effects of political communication", en Mass Communication and Society, vol. 3, núm. 2-3, Inglaterra: Routledge.

Sheafer, Tamir y Weimann, Gabriel (2005), "Agenda building, agenda setting, priming, individual voting intentions, and the aggregate results: An analysis of four Israeli elections", en Journal of Communication, vol. 55, núm. 2, Estados Unidos: Oxford University Press.

Shehata, Adam y Strömbäck, Jesper (2014), "Mediation of Political Realities: Media as Crucial Sources of Information”, en Esser, Frank y Strömbäck, Jesper [eds.], Mediatization of Politics, Reino Unido: Palgrave Macmillan.

So, Jiyeon et al. (2019), "Information seeking upon exposure to risk messages: predictors, outcomes, and mediating roles of health information seeking", en Communication Research, vol. 46, núm. 5, Estados Unidos: Sage.

Tai, Zixue y Sun, Tao (2007), "Media dependencies in a changing media environment: The case of the 2003 SARS epidemic in China”, en New Media and Society, vol. 9, núm. 6, Estados Unidos: Sage.

Todhunter, James (2013), “The domestic fruits of diplomacy: Mediation and presidential approval”, en International Negotiation, vol. 18, núm. 2, Holanda: Brill.

Wilkin, Holley y Ball-Rokeach, Sandra (2006), "Reaching at risk groups. The importance of health storytelling in Los Angeles Latino media”, en Journalism, vol. 7, núm. 3, Estados Unidos: Sage.

World Internet Stats (2021), “Digital 2021: Mexico”. Disponible en: https://datareportal. com/reports/digital-2021-mexico [28 de septiembre de 2021].

Yoo, Woohyun et al. (2020), "Differential effects of content-oriented versus user-oriented social media on risk perceptions and behavioral intentions", en Health Communication, vol. 35, núm. 1, Inglaterra: Routledge. 


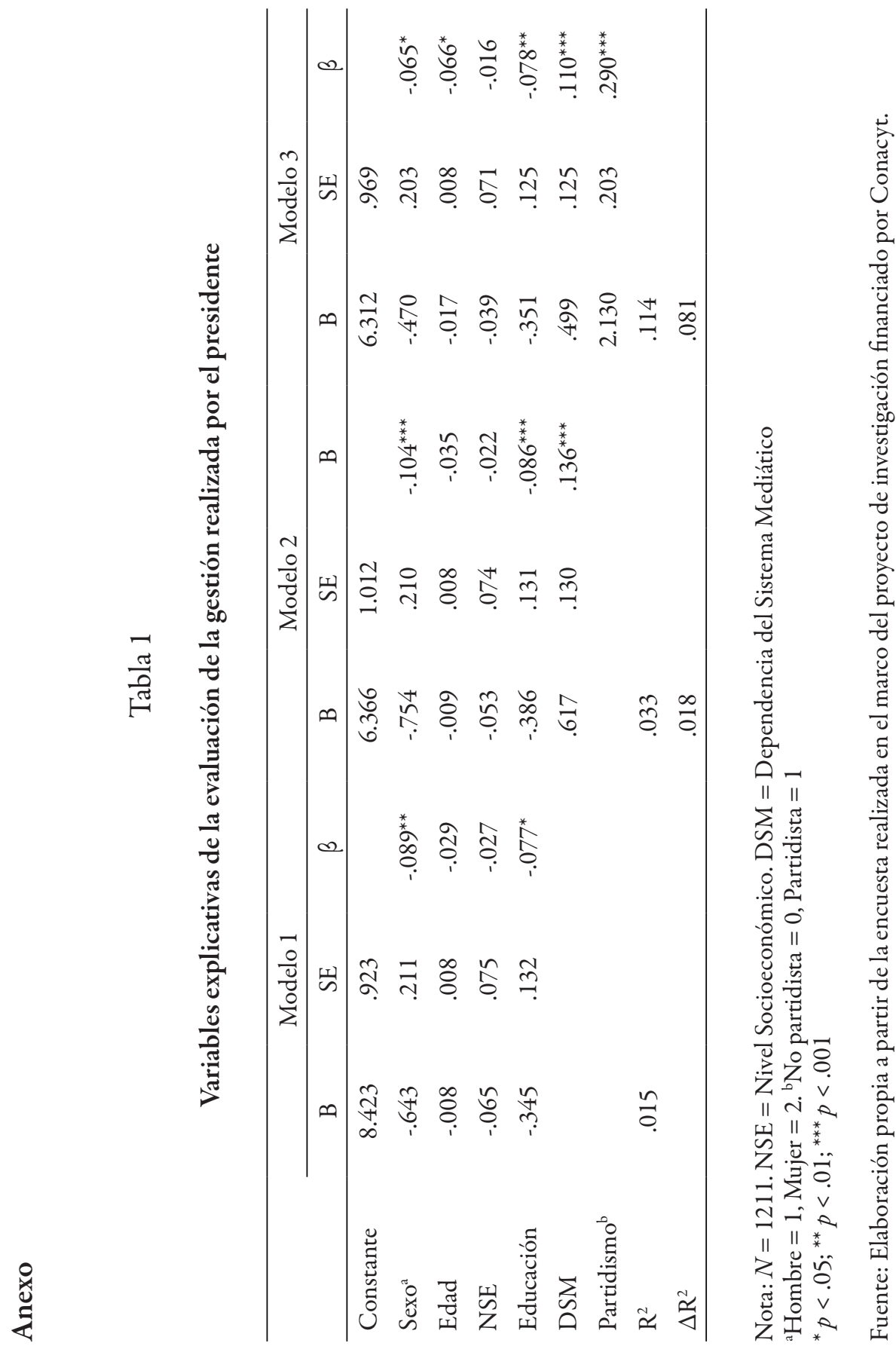




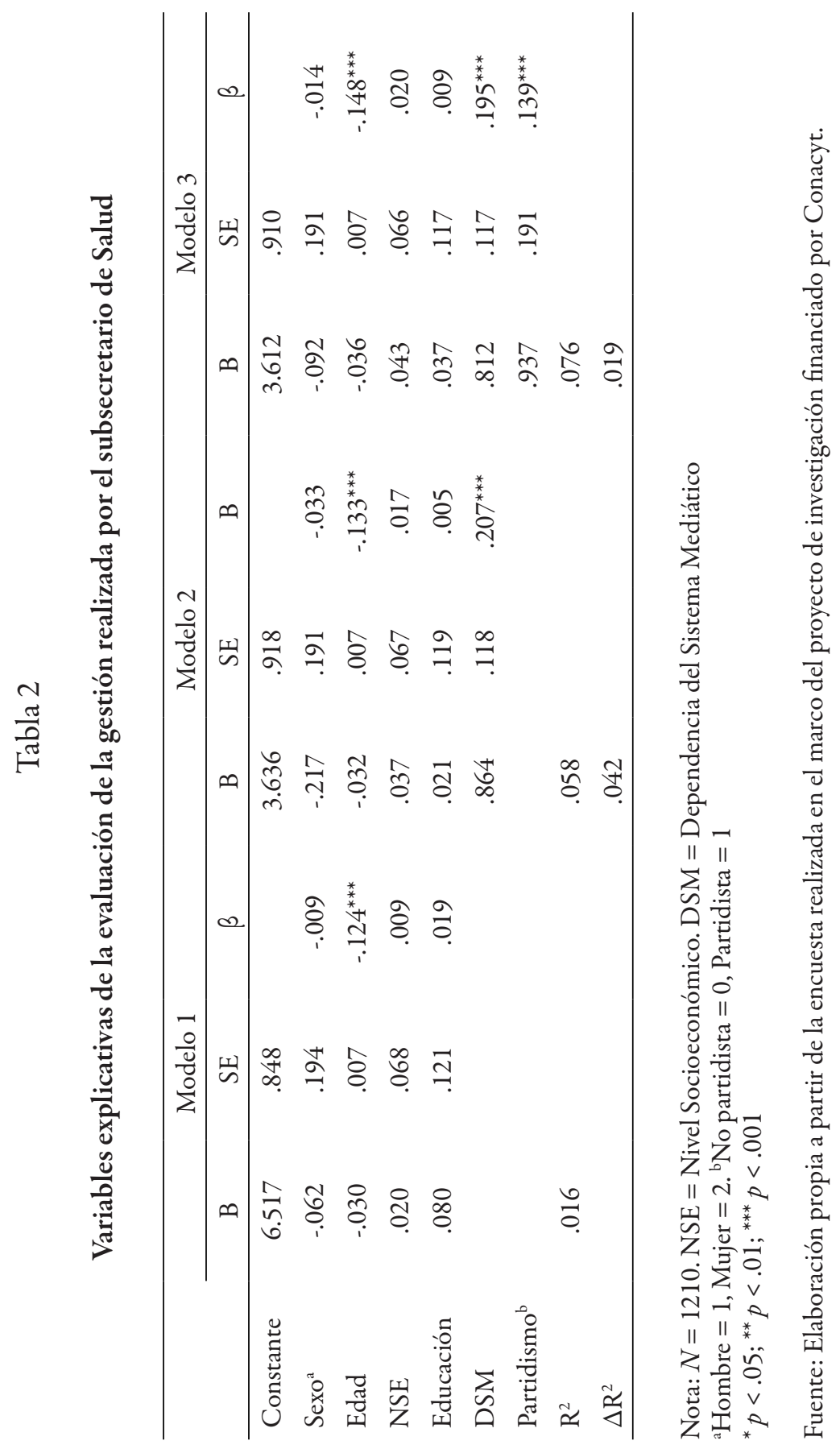




\section{Figura 1}

Comparativa de la evaluación de la gestión de ambos actores políticos

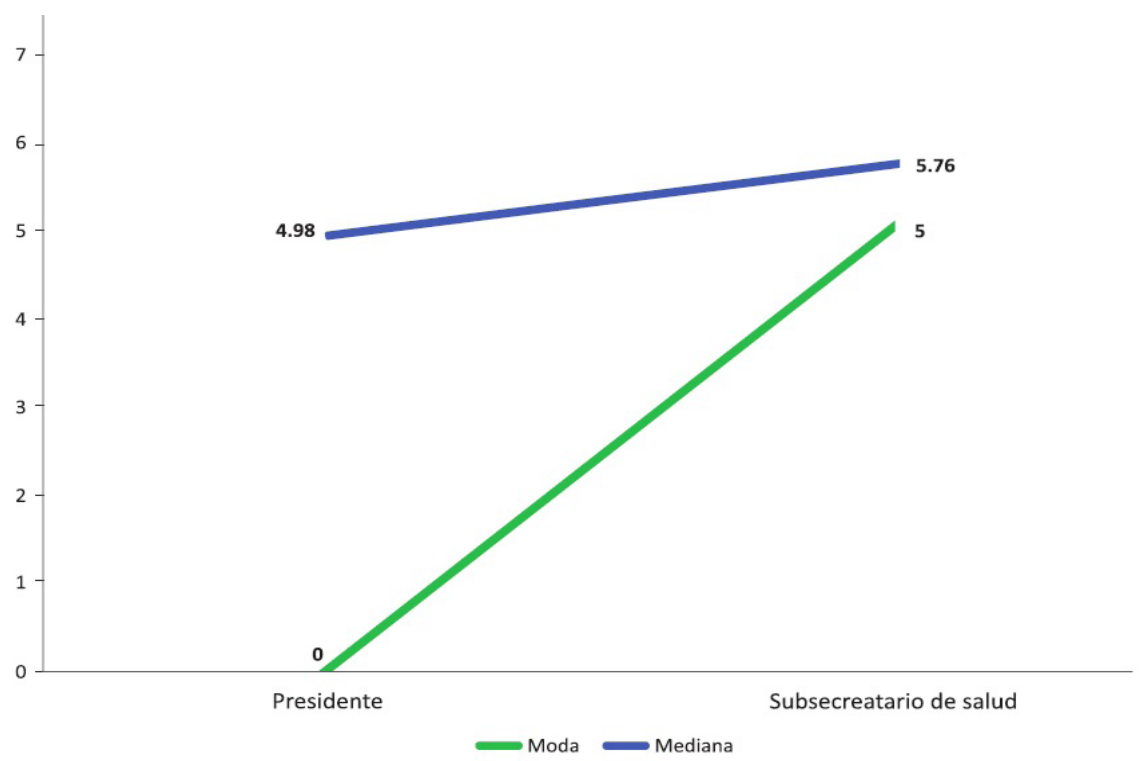

Fuente: Elaboración propia a partir de la encuesta realizada en el marco del proyecto de investigación financiado por Conacyt. 
Figura 2

Efecto moderador del partidismo en la evaluación de la gestión del presidente

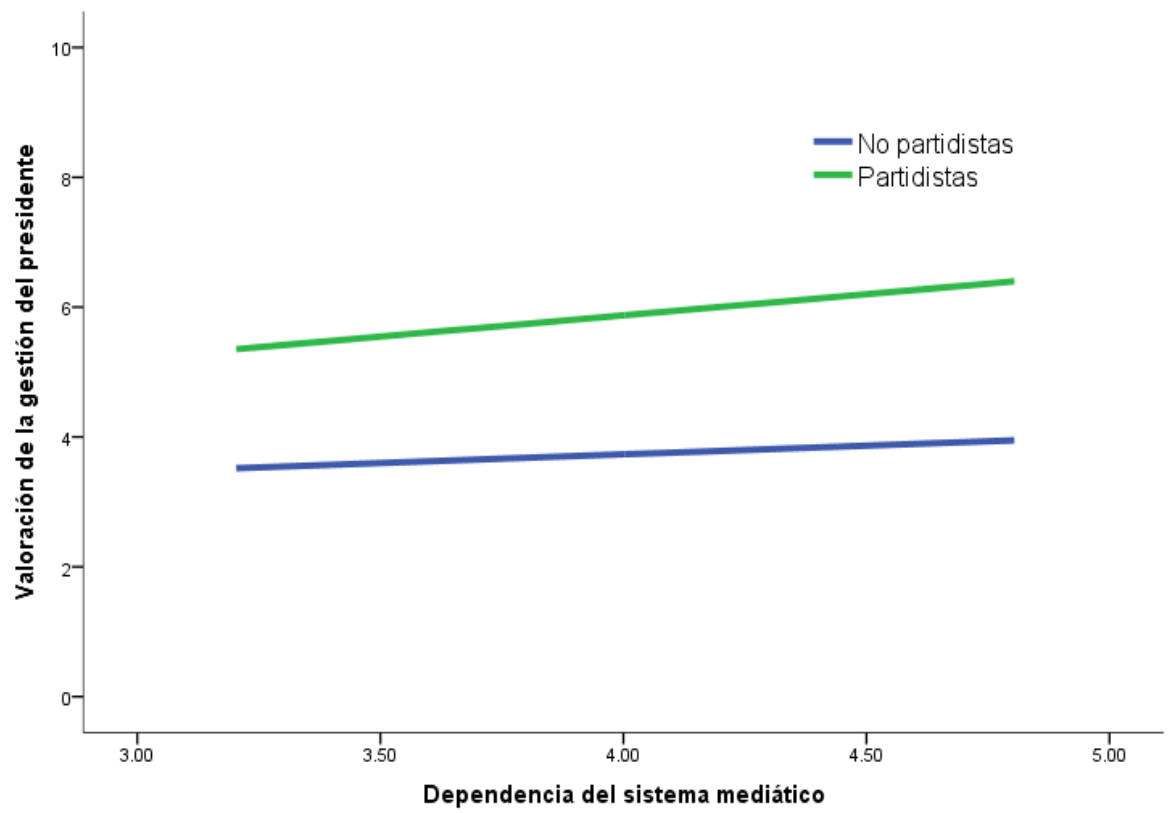

Fuente: Elaboración propia a partir de la encuesta realizada en el marco del proyecto de investigación financiado por Conacyt. 
Figura 3

Efecto moderador del partidismo en la evaluación de la gestión del subsecretario de Salud

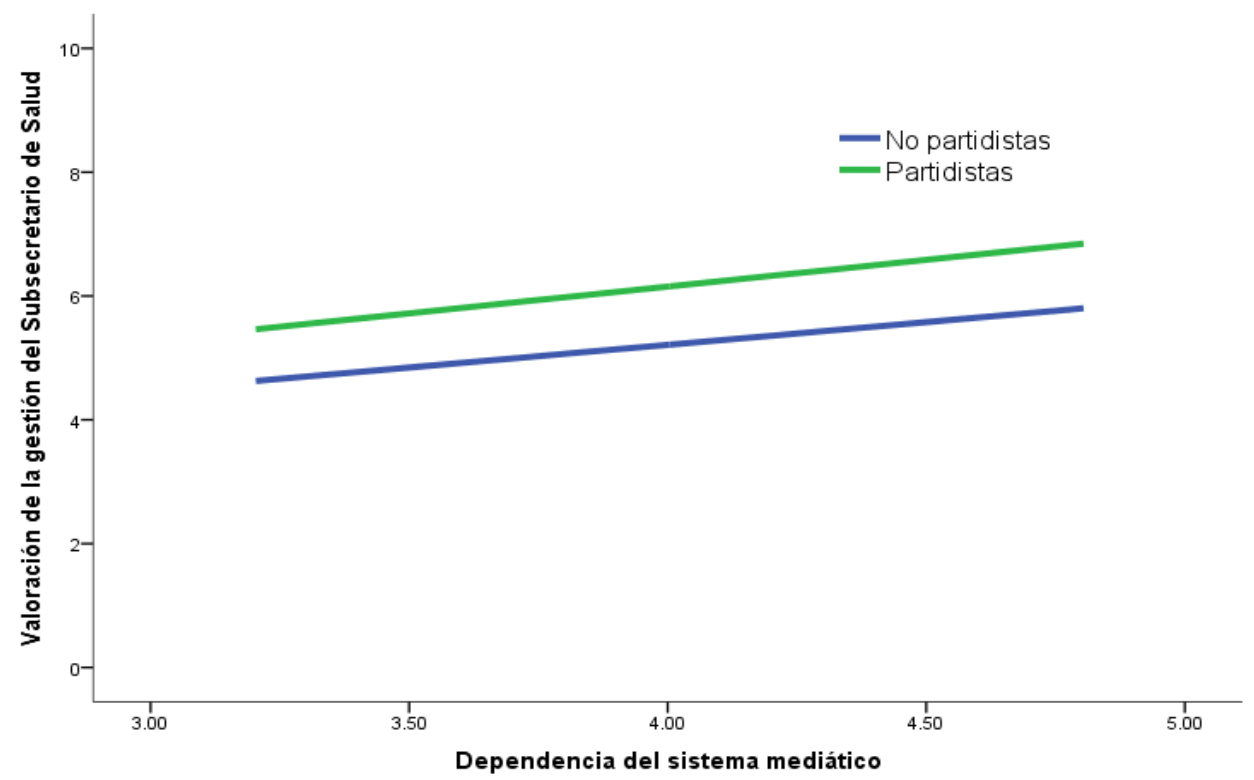

Fuente: Elaboración propia a partir de la encuesta realizada en el marco del proyecto de investigación financiado por Conacyt. 
Carlos Muñiz. Doctor en Comunicación. Profesor Titular en la Facultad de Ciencias Políticas y Relaciones Internacionales de la Universidad Autónoma de Nuevo León. Líneas de investigación: efectos mediáticos en el área de la comunicación política y tratamiento informativo (framing) de los acontecimientos. Es miembro del Sistema Nacional de Investigadores (SNI) en el nivel II. Publicaciones recientes: 1) Muñiz, Carlos (2021), "Construyendo la polarización a través del discurso político. Análisis del uso de claves periféricas en la retórica del presidente mexicano López Obrador", en Cultura, Lenguaje y Representación, vol. 26, España, Universidad Jaume I; 2) Muñiz, Carlos (2020), "El framing como proyecto de investigación: una revisión de los conceptos, ámbitos y métodos de estudio", en Profesional de la Información, vol. 29, núm. 6, España: Swets \& Zeitlinger; 3) Muñiz, Carlos (2020), "Media system dependency and change in risk perception during the COVID-19 pandemic", en Tripodos, vol. 1, núm. 47, España, Universidad Ramon Llull.

Victoria Isabela Corduneanu. Profesora-investigadora en la Academia de Comunicación y Cultura de la Universidad Autónoma de la Ciudad de México. Doctora en Historia y Civilización. Líneas de investigación: estudios de comunicación y juventud, y movimientos y movilizaciones sociales. Es miembro del Sistema Nacional de Investigadores (SNI) en el nivel I. Publicaciones recientes: 1) Muñiz, Carlos y Corduneanu, Victoria Isabela (2020), "Percepción de riesgo y consumo mediático durante el inicio de la pandemia de COVID-19 en México”, en Más Poder Local, núm. 41, España: Asociación Latinoamericana de Investigadores en Campañas Electorales; 2) Corduneanu, Victoria Isabela, Muñiz, Carlos y Echeverría, Martín (2019), "Emociones en contexto electoral y atención a política en medios: ¿ Inteligencia Afectiva o Evaluación Cognitiva?", en Perspectivas de la Comunicación, vol. 12, núm. 2, Chile: Universidad de La Frontera; 3) Corduneanu, Victoria Isabela (2019), "El papel de las emociones sociales y personales en la participación política”, en Revista Mexicana de Opinión Pública, núm. 26, México: Universidad Nacional Autónoma de México. 